\title{
Domesticity in generalized quadrangles
}

\author{
B. Temmermans* J. A. Thas H. Van Maldeghem \\ Department of Mathematics, Ghent University, \\ Krijgslaan 281, S22, B-9000 Gent \\ btemmerm@cage.UGent.be, jat@cage.UGent.be, hvm@cage.UGent.be
}

\begin{abstract}
An automorphism of a generalized quadrangle is called domestic if it maps no chamber, which is here an incident point-line pair, to an opposite chamber. We call it point-domestic if it maps no point to an opposite one and line-domestic if it maps no line to an opposite one. It is clear that a duality in a generalized quadrangle is always point-domestic and line-domestic.

In this paper, we classify all domestic automorphisms of generalized quadrangles. Besides three exceptional cases occurring in the small quadrangles with orders $(2,2)$, $(2,4)$ and $(3,5)$, all domestic collineations are either point-domestic or line-domestic. Up to duality, they fall into one of three classes: either they are central collineations, or they fix an ovoid, or they fix a large full subquadrangle. Remarkably, the three exceptional domestic collineatons in the small quadrangles mentioned above all have order 4 .
\end{abstract}

KEYwORDS: collineations, generalized polygons, opposition

MSC NuMBER: 51E12

${ }^{*}$ Supported by the Fund for Scientific Research - Flanders (FWO - Vlaanderen), Research Project G.0086.06 


\section{Introduction}

Brown \& Abramenko [2] show that every automorphism of an irreducible non-spherical building has infinite displacement. Their method also gives information about the spherical case. For instance, in the rank 2 case, every automorphism maps some chamber to a chamber at codistance at most one, and if the diameter of the incidence graph is even (odd), then any duality (collineation) maps some chamber to an opposite one. For generalized quadrangles, this shows that dualities behave normally, where 'normal' means that at least one chamber is mapped onto an opposite one. Counterexamples to this normal behaviour are given in [2], attributed to the third author of this paper, and consist of symplectic polarities in projective spaces and central collineations in generalized polygons of even diameter. The goal of this paper is to classify all 'abnormal' automorphisms of generalized quadrangles, which we will call 'domestic'.

The motivation for this work is to gather more qualitative information about general collineations in generalized quadrangles. When dealing with collineations, one usually considers fixed point structures, possibly using Benson's formulae [3], which gives a relation between the number of fixed points and those mapped onto collinear ones. The idea of the present paper is to consider also chambers and to offer a complementary look: what can we say about points, lines and chambers that are mapped onto opposite ones (which is complementary to the fixed ones). And it turns out that one can classify those collineations that have no points, lines or chambers like this at all. A useful consequence is that, given an arbitrary 'generic' collineation of a generalized quadrangle, it always maps some chamber to an opposite one.

A second motivation for this work is given by the result itself. It will turn out that, up to conjugation, there are precisely three domestic collineations which map some point to an opposite one, and which map some line to an opposite one. These three collineations all have order 4 and they act on small generalized quadrangles. Preliminary work on other generalized polygons shows that in the small cases there again exist such domestic collineations, and again they have order 4. However, it seems to be difficult to classify these exceptional domestic collineations in the case of generalized $n$-gons, with $n$ even and $n \geq 6$. In any case, it is somehow mysterious that all known examples of such exceptional domestic collineations have order 4 and only appear in small examples. 


\section{Preliminaries and statement of the Main Result}

We will write down most definitions in the general context of spherical buildings, but we will only be concerned with a specific class of buildings, namely, generalized quadrangles. Hence we do not define a building in full generality, but refer to the literature.

Let $\Omega$ be a spherical building, and let $\theta$ be an automorphism of $\Omega$. We emphasize that $\theta$ need not be type-preserving. Then we call $\theta$ domestic if no chamber of $\Omega$ is mapped onto an opposite chamber. More in particular, for a subset $J$ of the type set of $\Omega$, we say that $\theta$ is $J$-domestic, if $\theta$ does not map any flag of type $J$ onto an opposite one. The main result of Section 5 of [2], also proved earlier by Leeb [5], using entirely different methods, asserts that every automorphism of any (thick) spherical building is not $J$-domestic, for some type subset $J$. Hence being not $J$-domestic seems to be the rule, and so it is worthwhile to look at automorphisms which are $J$-domestic, for some $J$.

We now specialize to generalized quadrangles. Recall that a generalized quadrangle is a point-line incidence structure $\Gamma=(\mathcal{P}, \mathcal{L}, \mathrm{I})$ such that the incidence graph $(\mathcal{P} \cup \mathcal{L}, \mathrm{I})$ has diameter 4 and girth 8 . If there are (possibly infinite) parameters $s, t$ such that every line contains $s+1$ points and every point is incident with $t+1$ lines, then we say that $(s, t)$ is the order of $\Gamma$. If the valency of every vertex in the above graph is at least 3 (in which case we say that the quadrangle is thick), then $\Gamma$ automatically admits an order. Interchanging the roles of points $\mathcal{P}$ and lines $\mathcal{L}$ is called dualizing, and definitions or properties all have dual forms, obtained by dualizing.

In a generalized quadrangle, a flag is a point, a line or an incident point-line pair and a chamber is an incident point-line pair. An element (a point or a line) $x$ of a generalized quadrangle is opposite an element $y$ if the distance, measured in the incidence graph, between $x$ and $y$ is equal to 4 . Two chambers are called opposite if their points are opposite and their lines are opposite.

We will call a non-type preserving automorphism a duality and a type preserving automorphism a collineation, but as we mentioned before, we only have to consider collineations.

According to the terminology introduced above for spherical buildings, we will call a collineation which maps no point to an opposite point point-domestic, a collineation which maps no line to an opposite line line-domestic and a collineation which maps no chamber to an opposite chamber domestic. Also, if a collineation $\theta$ in a generalized quadrangle fixes all points collinear with a given point $x$, then we call $\theta$ a central collineation or a symmetry about the point $x$. Dually, we have axial collineations or symmetries about a line. We use common terminology such as 'collinear points', 'concurrent lines', etc. See [6] for more information and terminology on generalized quadrangles, and [14] for similar definitions 
on generalized polygons. Let us mention that an ovoid of a generalized quadrangle is a set $\mathcal{O}$ of points such that every line of the quadrangle is incident with exactly one element of $\mathcal{O}$. The dual notion is a spread. A full subquadrangle $\Gamma^{\prime}$ of a generalized quadrangle $\Gamma$ is a subquadrangle such that all points of $\Gamma$ of any line of $\Gamma^{\prime}$ are also points of $\Gamma^{\prime}$. The dual notion is an ideal subquadrangle.

The following theorem is our main result:

Theorem 2.1 If $\theta$ is a domestic collineation of a (not necessarily finite) thick generalized quadrangle $\Gamma$ of order $(s, t)$ then we have one of the following possibilities.

(i) $\theta$ is either point-domestic or line-domestic.

(ii) $(s, t) \in\{(2,2),(2,4),(4,2)\}, \theta$ is neither point-domestic nor line-domestic and $\theta$ has fixed elements; $\theta$ has a unique fixed chamber in case $(s, t)=(2,2)$, a unique fixed point and three fixed lines incident with it in case $(s, t)=(2,4)$, and the dual in the case $(s, t)=(4,2)$.

(iii) $(s, t) \in\{(3,5),(5,3)\}, \theta$ is neither point-domestic nor line-domestic, $\theta$ has no fixed elements and maps exactly 48 points to collinear points and 48 lines to concurrent lines.

Also, if $\theta$ is line-domestic, then we have one of the following possibilities.

(i) There are no fixed lines and the fixed points of $\theta$ form an ovoid.

(ii) There are fixed lines, but not two opposite ones. Then $\theta$ is a central collineation.

(iii) There are two opposite fixed lines and the fixed point-line structure is a full subquadrangle $\Gamma^{\prime}$ of $\Gamma$ with the additional property that every line off $\Gamma^{\prime}$ meets $\Gamma^{\prime}$ in a unique point. In the finite case this is equivalent with $\Gamma^{\prime}$ having order $(s, t / s)$.

And also, if $\theta$ is point-domestic, then we have one of the following possibilities.

(i) There are no fixed points and the fixed lines of $\theta$ form a spread.

(ii) There are fixed points, but not two opposite ones. Then $\theta$ is an axial collineation. 
(iii) There are two opposite fixed points and the fixed point-line structure is an ideal subquadrangle $\Gamma^{\prime}$ of $\Gamma$ with the additional property that every point off $\Gamma^{\prime}$ is incident with a unique line of $\Gamma^{\prime}$. In the finite case this is equivalent with $\Gamma^{\prime}$ having order $(s / t, t)$.

The subquadrangles in (iii) above are sometimes called large subquadrangles.

Let us mention a corollary to show the immediate usefulness of our result.

In Phan theory, see for instance [4], the Phan geometry of an involution of a (twin) building is the geometry induced on the chambers which are mapped onto opposite chambers. Our main result above characterizes all involutions of generalized quadrangles which have empty Phan geometry. Since Phan theory deals with Moufang buildings, we state our corollary in these terms, and refer to the literature for more information on Moufang quadrangles, see $[13,14]$. In particular, a Moufang quadrangle has a characteristic, which is the characteristic of any underlying field.

Corollary 2.2 Suppose the Phan geometry of an involution $\theta$ of a Moufang quadrangle $\Gamma$ is empty. Then $\theta$ fixes either a geometric hyperplane, or a dual geometric hyperplane. In particular, if the characteristic of $\Gamma$ is not equal to 2 , then either an ovoid, a spread, or a large subquadrangle is pointwise fixed.

In more specific cases, we can state this in more detail. For instance, exceptional quadrangles do not admit large subquadrangles, and we conjecture that an involution in such a quadrangle can never fix an ovoid or a spread (this conjecture is supported by the fact that the existence of such a fixed ovoid or spread would imply the existence of a yet unknown Moufang set of algebraic nature, which is highly unlikely). Also, the geometric hyperplanes of the embeddable Moufang quadrangles (the 'classical' ones) are obtained by intersecting with a hyperplane of the ambient projective space related to the 'universal embedding'.

Before embarking on the proof of Theorem 2.1, we comment on the situation in other types of buildings. For projective spaces, domestic collineations and dualities are completely classified in [9]. The result is that the only domestic duality is a symplectic polarity, and a collineation in an $n$-dimensional projective space is domestic if and only if it pointwise fixes a subspace of dimension at least $\frac{n+1}{2}$. For polar spaces, there is only a full classification available in the case of rank 3. This, and partial results in arbitrary rank, can be found in [10]. For other types of spherical buildings, nothing is known, except for generalized $n$-gons with $n$ odd, where one can show that no domestic collineation or dualities exist at all (see [7]) . 


\section{The generic case}

We shall prove Theorem 2.1 in a sequence of lemmas and propositions. In this section, we will focus on the generic case. So we exclude the small cases that will provide the exceptional examples. However, the small examples will be excluded in different circumstances, and this will enable us to give some general information about the exceptional cases, which we will then use in the next section.

Throughout, let $\theta$ be a domestic collineation of a generalized quadrangle $\Gamma$ with order $(s, t)$. Our first big aim is to prove the following proposition.

Proposition 3.1 If $(s, t) \notin\{(2,2),(2,4),(4,2),(3,5),(5,3)\}$, then $\theta$ is either point-domestic or line-domestic. Also, if $\theta$ is neither point-domestic nor line-domestic and has fixed elements, then $(s, t) \in\{(2,2),(2,4),(4,2)\}$. If $\theta$ is neither point-domestic nor line-domestic and has no fixed elements, then $(s, t) \in\{(3,5),(5,3)\}$.

We will prove this proposition in a few lemmas.

Lemma 3.2 Suppose that $t \geq 3$, and that $s \geq 7$. If a line $X$ is mapped onto an opposite line $X^{\prime}$, then $\left\{X, X^{\prime}\right\}^{\perp}$ is fixed elementwise. Also, $\theta$ is point-domestic.

Proof Suppose a line $X$ is mapped onto an opposite line $X^{\prime}$ and some element $Y$ concurrent with both $X, X^{\prime}$ is not fixed. We note that every point $p$ on $X$ is mapped onto $\operatorname{proj}_{X^{\prime}} p$. Hence the intersection $x:=X \cap Y$ is mapped onto $x^{\prime}:=X^{\prime} \cap Y$, and so $Y^{\theta}$ is incident with $x^{\prime}$. We assume, by way of contradiction, that $Y^{\theta} \neq Y$. Hence, every point on $Y$ distinct from $x$ and from $x^{\prime}$ is mapped onto an opposite point. Let $y$ be such a point. Since $\theta$ is domestic, every line through $y$ is mapped onto a concurrent line. Choose three such lines $L_{1}, L_{2}, L_{3}$, all distinct from $Y$. On $L_{i}, i \in\{1,2,3\}$, there is a unique point $z_{i}$ that is mapped onto the intersection $L_{i} \cap L_{i}^{\theta}$ (and since $y^{\theta} \neq y$, we have $L_{i} \neq L_{i}^{\theta}$, so that the intersection is well defined). Let $z_{i}^{\prime}$ be the projection of $z_{i}$ onto $X, i \in\{1,2,3\}$. Note that, applying $\theta$, $\operatorname{proj}_{X^{\prime}} z_{i}^{\prime}$ is collinear to both $x^{\theta}=x^{\prime}$ and $z_{i}^{\theta}$. Since $s \geq 7$, there exists a point $w$ on $X$ distinct from $x, z_{1}^{\prime}, z_{2}^{\prime}, z_{3}^{\prime}, \operatorname{proj}_{X} z_{1}^{\theta}, \operatorname{proj}_{X} z_{2}^{\theta}, \operatorname{proj}_{X} z_{3}^{\theta}$. Let $w_{i}$ be the projection of $w$ onto $L_{i}, i \in\{1,2,3\}$. Then $w_{i}^{\theta}$ is opposite $w_{i}$. Hence the image of the line $w w_{i}, i=1,2,3$, is concurrent with the line $w w_{i}$, contradicting the fact that at most two lines through $w$ are mapped onto a concurrent one (namely, the line through $w$ and $w^{\theta}$, and the preimage of that line).

Suppose now that some point $v$ is mapped onto an opposite point $v^{\theta}$. Let $v^{\prime}$ be the projection of $v$ onto $X$. Since $v^{\prime}$ is mapped onto a collinear but distinct point, and since 
$v v^{\prime}$ is not fixed, we see that $v v^{\prime}$ is mapped onto an opposite line, contradicting domesticity of $\theta$.

This lemma together with its dual proves Proposition 3.1 except for the cases $(s, t) \in$ $\{(2,2),(2,4),(3,3),(3,5),(4,4),(4,6),(5,5),(6,6)\}$ (up to duality).

The following lemma makes some progress for the bigger values of $s$ and $t$ in the foregoing list, and shows that there are no fixed elements in the cases $(s, t) \in\{(3,5),(5,3)\}$.

Lemma 3.3 Suppose that $\Gamma$ is finite and that $\theta$ has fixed elements. Suppose also that $\theta$ is neither point-domestic nor line-domestic. Then either $s=2$ or $t=2$. If $(s, t)=(2,2)$, then $\theta$ has a unique fixed point and a unique fixed line (both incident with each other). If $(s, t)=(4,2)$, then $\theta$ fixes a unique line and exactly three points incident with that line.

Proof Suppose $\theta$ is neither point-domestic nor line-domestic and suppose that $\theta$ fixes at least one point $x$. Then there exists a line $X$ which is mapped onto an opposite line $X^{\prime}$. Because the projection $x^{\prime}:=\operatorname{proj}_{X} x$ is collinear with its image $x^{\prime \theta}$, it is easy to see that $x$ is incident with the line $Y:=x^{\prime} x^{\prime \theta}$. Hence the line $Y$ is fixed (which will allow us below to use the dual arguments of what follows). Suppose first that all lines concurrent with $X$ and $X^{\prime}$ are fixed. We assumed that $\theta$ is not point-domestic, hence there exists a point $p$ which is mapped to an opposite point. This point can not lie on one of the fixed lines which are concurrent with $X$ and $X^{\prime}$. Consider the point $p^{\prime}:=\operatorname{proj}_{X} p$. The line $p p^{\prime}$ would be mapped to an opposite line and we obtain a flag $\left\{p, p p^{\prime}\right\}$ which is mapped to an opposite flag, a contradiction. Hence we can assume that there is a line $Z$, concurrent with $X$ and $X^{\prime}$ which is not fixed. Because the points on this line, not incident with $X$ or $X^{\prime}$ are mapped to opposite points, it follows that every point $y$ on $Y$, not incident with $X$ or $X^{\prime}$, is fixed. Otherwise, the flag $\left\{\operatorname{proj}_{Z} y,\left\langle y, \operatorname{proj}_{Z} y\right\rangle\right\}$ would be mapped to an opposite flag.

If $s=2$, then we know that $t \in\{2,4\}$ and all lines are regular. This implies that exactly one line meeting both $X$ and $X^{\prime}$ is fixed, and so, there is a unique fixed point. If $(s, t)=(2,2)$, then also the dual holds and we obtain a unique fixed point and a unique fixed line, incident with one another.

If $(s, t)=(4,2)$, then the duals of the previous arguments imply that there is a unique fixed line, which can be taken as $Y$. Then $Y$ contains exactly 3 fixed points. As there are no further fixed lines, there are neither further fixed points.

From now on, we may assume that $s \geq 3$ and $t \geq 3$ and we aim for a contradiction. Hence there exists a line $M$ through $X \cap Z$ different from $X, Z$ and $Z^{\theta^{-1}}$. This line is mapped 
to an opposite line through $X^{\prime} \cap Z$. Let $y^{\prime}$ be the projection of a point $y$ of $Y$ onto $M$. The image of $y^{\prime}$ is a point $y^{\prime \theta}$ on $M^{\theta}$ which is collinear to $y^{\prime}$. Hence the line $y y^{\prime}$ is fixed. By equivalent reasons as before every point $z$ on $y y^{\prime}$ different from $y^{\prime}$ and $y^{\prime \theta}$ is fixed. If $z^{\prime}$ is the point of $X$ collinear with $z$, then $z z^{\prime} \in\left\{X, X^{\prime}\right\}^{\perp}$ is fixed. Hence exactly $s-1 \geq 2$ lines of $\left\{X, X^{\prime}\right\}^{\perp}$ are fixed under $\theta$. Let $U$ be one of these, $U \neq Y$.

If a line $L$ through $x$ is not fixed, then all points of $L$ except for $x$ are mapped onto opposites. Hence, since $\theta$ is domestic, the line $U^{\prime}$ through $X \cap U$ and concurrent with $L$ must be mapped onto a concurrent line. Since $U$ is fixed, $U^{\prime}$ must necessarily coincide with $U$. Hence $U^{\prime}$ is fixed, and so is $L$, a contradiction.

Because $x$ was an arbitrary fixed point, it follows that every line through every fixed point is fixed. Hence we obtain a fixed subquadrangle of order $(s-2, t)$. Dually, this subquadrangle must also have order $(s, t-2)$, a contradiction.

Lemma 3.4 Suppose that $(s, t) \in\{(2,2),(2,4),(3,3),(4,2),(4,4),(4,6),(5,5),(6,4),(6,6)\}$ and that there are no fixed elements, then $\theta$ is the identity.

Proof If by way of contradiction $\theta$ is not the identity, then there is at least one element which is mapped to an opposite. So up to duality we may assume that there exists a line $X$ which is mapped onto an opposite line $X^{\prime}$. We will first count the number of points which are mapped to a collinear point. For every point $p$ on $X$ there are $t-1$ lines through $p$ (including $X$ ) which are mapped to opposite lines. Hence all the points on these lines should be mapped to collinear points. On each of the two remaining lines through $p$, there are exactly two points which are mapped to collinear ones (twice including $p$ ). Hence $2(s-1)$ points collinear to $p$ are mapped to opposite ones. Hence there are $(s+1) 2(s-1)$ points which are mapped to opposite points and $(s+1)(t-2) s+3(s+1)$ points which are mapped to collinear points. Dually the number of lines mapped to opposite lines is equal to $2\left(t^{2}-1\right)$. We will now calculate this number in a different way.

We count the number of flags $\{y, Y\}$ which are mapped to a flag $\{y, Y\}^{\theta}$ where $Y$ and $Y^{\theta}$ are opposite lines and $y$ and $y^{\theta}$ are collinear points. Suppose that $n$ is the number of lines which are mapped to an opposite line. Then there are $n(s+1)$ such flags. We can also count these flags as follows. There are $(s+1)(t-2) s+3(s+1)$ points which are mapped to a collinear point, through each of these points there are $t-1$ lines which are mapped to an opposite line. Hence $n(s+1)$ should be equal to $((s+1)(t-2) s+3(s+1))(t-1)$ and hence $n=((t-2) s+3)(t-1)$.

Combining the two previous paragraphs we obtain $3 t+2 s+s t^{2}=3 s t+2 t^{2}+1$. If $(s, t) \in$ $\{(2,2),(2,4),(3,3),(4,2),(4,4),(4,6),(5,5),(6,4),(6,6)\}$, we obtain a contradiction. 
The last identity of the previous proof is fulfilled for both $(s, t)=(3,5)$ and $(s, t)=(5,3)$. This is rather surprising, as the identity is far from being symmetric in $s$ and $t$. Yet, the right exceptions emerge! Moreover, the previous proof shows that a domestic collineation in a quadrangle of order $(3,5)$ which is neither point- nor line domestic must have exactly $(s+1)(t-2) s+3(s+1)=48$ points mapped to a collinear point. This observation completes the proof of the fact that domestic collineations of generalized quadrangles which are neither point-domestic nor line-domestic can only exist for orders $(2,2),(2,4)$ and $(3,5)$, up to duality. Moreover, taking account of Lemma 3.3, in the first case there is a unique fixed chamber, in the second case there is a unique fixed point and exactly three fixed lines, and in the last case there are no fixed elements and precisely 48 points mapped onto collinear ones and hence also 48 lines mapped to concurrent ones.

Now we investigate what happens if $\theta$ is line-domestic.

Lemma 3.5 If no line is mapped onto an opposite line, then we have one of the following possibilities.

(i) There are no fixed lines and the fixed points of $\theta$ form an ovoid.

(ii) There are fixed lines, but not two opposite ones. Then $\theta$ is a central collineation.

(iii) There are two opposite fixed lines and the fixed point-line structure is a full subquadrangle $\Gamma^{\prime}$ of $\Gamma$ with the additional property that every line off $\Gamma^{\prime}$ meets $\Gamma^{\prime}$ in a unique point. In the finite case this is equivalent with $\Gamma^{\prime}$ having order $(s, t / s)$.

Proof Suppose a line $L$ is not fixed. Then there is a unique point $x$ in the intersection of $L$ and $L^{\theta}$. If $x$ were not fixed, then every line distinct from $L$ and $L^{\theta}$ and incident with $x$ would be mapped onto an opposite line, a contradiction. Hence $x$ is fixed.

So, if no line is fixed, then every line is incident with a unique fixed point, and hence these form an ovoid.

Suppose now that there is at least one fixed line $L$ and all fixed lines are concurrent and incident with some point $z$. Note that every point on any fixed line is a fixed point, as otherwise some line concurrent with that fixed line is mapped onto an opposite line. Suppose now that some line $M$ concurrent with $L$ is not fixed. Then every point $x$ on $M$, not on $L$, is mapped onto an opposite point $x^{\theta}$, and so every line through $x$ is mapped onto a concurrent line. This implies the existence of a fixed point $u$ off $L$, and hence of a fixed line $L^{\prime}$ different from $L$, but concurrent with it in the point $z$ (by assumption). Note that $M$ cannot be incident with $z$, as $x$ and $x^{\theta}$ are both collinear with $u$, and $u$ is 
collinear with $z$. In particular, it follows that all lines through $z$ are fixed, and that we have a central collineation with center $z$.

If $L$ and $M$ are two opposite fixed lines, then, in view of the fact that every point on every fixed line is fixed, we see that the fixed point structure is a full subquadrangle $\Gamma^{\prime}$. If some line off $\Gamma^{\prime}$ did not meet $\Gamma^{\prime}$, then it would be mapped onto an opposite line (as otherwise the intersection with its image is fixed and belongs to $\Gamma^{\prime}$ by definition of $\Gamma^{\prime}$ ). In the finite case, it follows from [11] and [12] that $\Gamma^{\prime}$ has order $(s, t / s)$.

The lemma is proved.

Combining the previous lemmas and their duals, we see that Theorem 2.1 is proved, up to the exceptional cases, which we will treat in the next section.

\section{Domestic collineations of the generalized quadran- gles of order $(2,2),(2,4)$ and $(3,5)$}

The restrictions that we assume are justified by arguments in the previous section. It turned out that these are the only cases in which domestic collineations that are neither point-domestic nor line-domestic can exist (we call these exceptional domestic collineations).

The methods that we use are far from uniform. We usually just pick a convenient description of the quadrangle in question and start arguing. The fact that all exceptional domestic collineations have order 4 suggests that there might be a uniform approach, but so far we have not been able to find one. Concerning the orders $(2,2)$ and $(2,4)$, our previous results imply that exceptional domestic collineations must live in the stabilizer of a flag, which is in both cases a 2-group, and so this hints at order 4 (as no involution can have the given fixed point structure). We could use this in the proof of the next lemma, but a direct approach is not much longer and provides an explicit description.

Lemma 4.1 Suppose $\Gamma$ has order $(2,2)$, then up to conjugation there is exactly one domestic collineation which is neither point-domestic nor line-domestic and which fixes exactly one point and one line. It has order 4.

Proof We will use the following model of the generalized quadrangle of order $(2,2)$, see Chapter 6 of [6]. The points are pairs $\{i, j\}, i \neq j$ and $i, j \in\{1,2,3,4,5,6\}$. The lines are partitions $\{\{i, j\},\{k, l\},\{m, n\}\}$, where $\{i, j, k, l, m, n\}=\{1,2,3,4,5,6\}$. The incidence 
relation is given by inclusion. The full automorphism group is given by the natural action of the symmetric group $\mathrm{S}_{6}$.

By assumption, we may assume that $\theta$ fixes the point $\{5,6\}$. It is easy to see that the only permutations in $\mathrm{S}_{6}$ fixing the pair $\{5,6\}$ and not any other pair, are conjugate to

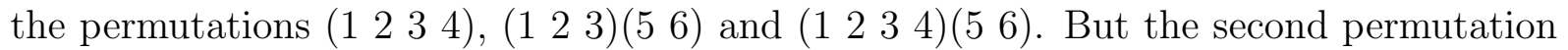
does not fix any line (contradicting our assumption), while the first one maps the flag $\{\{1,2\},\{\{1,2\},\{3,5\},\{4,6\}\}\}$ to the opposite flag $\{\{2,3\},\{\{2,3\},\{4,5\},\{1,6\}\}\}$. Also, one easily checks that (1 2334$)\left(\begin{array}{lll}5 & 6\end{array}\right)$ satisfies the given conditions.

Lemma 4.2 Suppose $\Gamma$ has order $(2,4)$, then up to conjugation there is exactly one domestic collineation which is neither point-domestic nor line-domestic and which fixes exactly one point and three lines. It has order 4.

Proof We will use the following model of the generalized quadrangle of order $(2,4)$, see Chapter 6 of [6]. The points are pairs $\{i, j\}$, with $i \neq j$ and $i, j \in\{1,2,3,4,5,6\}$, and the symbols $1,2,3,4,5,6$ and $1^{\prime}, 2^{\prime}, 3^{\prime}, 4^{\prime}, 5^{\prime}, 6^{\prime}$. The lines are triples $\{\{i, j\},\{k, l\},\{m, n\}\}$, where $\{i, j, k, l, m, n\}=\{1,2,3,4,5,6\}$, and triples $\left\{i,\{i, j\}, j^{\prime}\right\}$, where $i, j \in\{1,2,3,4,5,6\}$, $i \neq j$. Incidence is given by inclusion.

By assumption, we know that $\theta$ fixes exactly one point and three lines incident with that point. So we may assume that the point $\{5,6\}$ is fixed, and that also the lines $\left\{5,\{5,6\}, 6^{\prime}\right\},\left\{6,\{5,6\}, 5^{\prime}\right\}$ and $\{\{5,6\},\{1,3\},\{2,4\}\}$ are fixed. Then $\theta$ interchanges the points 5 and $6^{\prime}$ and the points 6 and $5^{\prime}$. We now claim that the size of the orbit of $\{1,2\}$ under $\theta$ is 4 . If not, then $\theta^{2}$ fixes all points of $\{5,6\}^{\perp}$. Take a point $x \notin\{5,6\}^{\perp}$. Then $x^{\theta^{2}}$ is collinear with all points of $\{\{5,6\}, x\}^{\perp}$, hence $x=x^{\theta^{2}}$ and hence $\theta^{2}=1$. Now take a line $L$ on $\{1,2\}$ which is not incident with $\{5,6\}$. The image $L^{\theta}$ of $L$ is incident with the point $\{1,2\}^{\theta}$ which is opposite $\{1,2\}$, hence, because $\theta$ is domestic, the lines $L$ and $L^{\theta}$ are concurrent. If $y$ is the intersection of $L$ and $L^{\theta}$, then it follows that $y^{\theta}=y$, a contradiction. Consequently we may assume, possibly by substituting $\theta$ with its inverse, that

$$
\theta:\{1,2\} \mapsto\{2,3\} \mapsto\{3,4\} \mapsto\{1,4\} \mapsto\{1,2\} .
$$

But now $\theta$ is completely determined, as every point $x$ opposite $\{5,6\}$ is itself determined by the trace $\{\{5,6\}, x\}^{\perp}$. For instance, the point 1 is collinear with $\left\{6^{\prime}, 5^{\prime},\{1,3\},\{1,2\},\{1,4\}\right\}$ which is mapped to $\{5,6,\{2,4\},\{2,3\},\{1,2\}\}$, and this set equals $\left\{\{5,6\}, 2^{\prime}\right\}^{\perp}$. One obtains that $\theta$ is naturally induced by the permutation $\varphi:=\left(\begin{array}{lll}1 & 2 & 3\end{array}\right)\left(\begin{array}{ll}5 & 6\end{array}\right)$ (in the sense that the image of the point $\{a, b\}, a \neq b$, under $\theta$ is the point $\left\{a^{\varphi}, b^{\varphi}\right\}$, and the images of the points $a$ and $b^{\prime}$ under $\theta$ are $\left(a^{\varphi}\right)^{\prime}$ and $b^{\varphi}$, respectively, for all $\left.a, b \in\{1,2, \ldots, 6\}\right)$, and 
one observes that the collineation induced by the permutation $(24)$ conjugates $\theta$ into its inverse. One easily checks that $\theta$ satisfies the conditions, which completes the proof of the lemma.

The following lemma belongs to folklore, but we provide a short proof for the sake of completeness.

Lemma 4.3 Let $\mathcal{O}$ be a hyperoval in $\mathrm{PG}(2,4)$. Let $\theta$ be a collineation of $\mathrm{PG}(2,4)$ preserving $\mathcal{O}$. Then the companion field automorphism of $\theta$ is trivial if and only if the permutation induced on $\mathcal{O}$ by $\theta$ is even.

Proof It is well known that the stabilizer of $\mathcal{O}$ in $\mathrm{P} \Gamma L_{3}(4)$ is isomorphic to the symmetric group $S_{6}$, with natural action on $\mathcal{O}$. Since $S_{6}$ has a unique subgroup of index 2 - which is the alternating group $A_{6}$ - and since the intersecion of $S_{6}$ with the subgroup $\mathrm{PGL}_{3}(4)$ of index 2 of $\mathrm{P} \Gamma \mathrm{L}_{3}(4)$ is a subgroup of index at most 2 in $\mathrm{S}_{6}$, it suffices to prove that at least one element of $S_{6}$ does not belong to $\mathrm{PGL}_{3}(4)$. But this is easy: any collineation of PG $(2,4)$ inducing a transposition on $\mathcal{O}$ fixes pointwise a quadrangle, and hence must be a Baer involution.

The lemma is proved.

Lemma 4.4 Suppose $\Gamma$ has order $(3,5)$, then up to conjugation there is exactly one domestic collineation which is neither point-domestic nor line-domestic, which has no fixed elements and for which exactly 48 points are mapped onto collinear points and 48 lines are mapped onto concurrent lines. It has order 4.

Proof We use the following model of the generalized quadrangle of order $(3,5)$, see Chapter 6 of [6]. The points are the points of a 3 -dimensional affine space AG $(3,4)$ over $\mathrm{GF}(4)$. The lines are the lines of $\mathrm{AG}(3,4)$ which meet the plane at infinity $\pi$ in a point of a fixed hyperoval $\mathcal{O}$. Introducing coordinates $X_{1}, X_{2}, X_{3}, X_{4}$ in the projective completion $\mathrm{PG}(3,4)$ of $\mathrm{AG}(3,4)$, we may assume that $\pi$ has equation $X_{4}=0$, and that the coordinates of the points of the hyperoval $\mathcal{O}$ are $(1,0,0,0),(0,1,0,0),(0,0,1,0),(1,1,1,0),\left(1, \epsilon, \epsilon^{2}, 0\right)$ and $\left(1, \epsilon^{2}, \epsilon, 0\right)$, where $\operatorname{GF}(4)=\left\{0,1, \epsilon, \epsilon^{2}\right\}$.

Suppose $\theta$ satisfies the assumptions of the lemma. By [1, Section 3], $\theta$ is induced by a collineation of $\mathrm{PG}(3,4)$ stabilizing $\mathcal{O}$. Hence $\theta$ induces a permutation of $\mathcal{O}$. If it fixes some point $x$ of $\mathcal{O}$, then, since $\theta$ does not fix any line of $\Gamma$, and the lines through $x$ are mutually opposite, $\theta$ must map every point to a collinear point, a contradiction. 
Hence $\theta$ induces a fixed point free permutation on $\mathcal{O}$. Since it also acts fixed point freely on $\mathrm{AG}(3,4)$ without cycles of length 2 (such a cycle of collinear points would imply a fixed line, such a cycle of non-collinear points would imply that all points collinear to both of these are fixed), the action on $\mathcal{O}$ must have order 2 or 4 .

Suppose first that the action of $\theta$ on $\mathcal{O}$ has order 4 . Then because of the foregoing lemma the companion field automorphism of $\theta$ is the identity (indeed, the action on $\mathcal{O}$ is an even permutation). So, up to conjugation, the matrix of $\theta$ has the form

$$
\left(\begin{array}{llll}
0 & 1 & 0 & 0 \\
0 & 0 & 1 & 0 \\
1 & 1 & 1 & 0 \\
a & b & c & d
\end{array}\right)
$$

If $d \neq 1$, then this matrix has an eigenvector belonging to the eigenvalue $d$, which gives rise to a fixed point in $\Gamma$. Hence $d=1$. Let $L$ be a line of $\Gamma$ containing the point $\left(1, \epsilon, \epsilon^{2}, 0\right)$ at infinity. Then this line contains at most one point $x$ with the property that $x^{\theta}$ belongs to $L$. Since by the observation just after Lemma 3.4 the collineation $\theta$ maps 48 points to collinear points, there are at most 32 points $x$ with $x x^{\theta}$ a line of $\Gamma$ through one of $\left(1, \epsilon, \epsilon^{2}\right)$ and $\left(1, \epsilon^{2}, \epsilon\right)$. Consequently, there is at least one point $y$ with $y y^{\theta}$ a line of $\Gamma$ through $(1,0,0,0)$. We may choose the coordinates of $y$ equal to $(0,0,0,1)$, and those of $y^{\theta}$ equal to $(1,0,0,1)$. Hence $a=d=1$ and $b=c=0$ in the above matrix. But now the flag $\{(1,0,0, \epsilon), M\}$, with $M$ the line through $(1,0,0, \epsilon)$ and $(0,1,0,0)$, is mapped onto the opposite flag $\left\{\left(1, \epsilon^{2}, 0,1\right), M^{\theta}\right\}$, with $M^{\theta}$ the line through $\left(1, \epsilon^{2}, 0,1\right)$ and $(0,0,1,0)$.

Hence $\theta$ induces a fixed point free involution on $\mathcal{O}$. It follows that the companion field automorphism is nontrivial this time, and we may assume that the matrix of $\theta$ has the form

$$
\left(\begin{array}{llll}
1 & 1 & 1 & 0 \\
0 & 0 & 1 & 0 \\
0 & 1 & 0 & 0 \\
a & b & c & d
\end{array}\right)
$$

We may assume that the point $(0,0,0,1)$ is mapped onto a collinear point, for which we may take without loss of generality the coordinates $(1,0,0,1)$. Hence $a=d$ and $b=c=0$. The cases $d=\epsilon$ and $d=\epsilon^{2}$ are equivalent by conjugating with the map that squares each coordinate of every point. But if $a=d=\epsilon$, then $\theta$ would map the flag

$$
\{(\epsilon, 0,0,1),\langle(\epsilon, 0,0,1),(0,1,0,0)\rangle\}
$$


onto the opposite flag

$$
\left\{\left(1, \epsilon^{2}, \epsilon^{2}, \epsilon\right),\left\langle\left(1, \epsilon^{2}, \epsilon^{2}, \epsilon\right),(0,0,1,0)\right\rangle\right\}
$$

a contradiction. Hence $\theta$ is given by

$$
\theta:\left(x_{1}, x_{2}, x_{3}, x_{4}\right) \mapsto\left(x_{1}^{2}, x_{2}^{2}, x_{3}^{2}, x_{4}^{2}\right)\left(\begin{array}{cccc}
1 & 1 & 1 & 0 \\
0 & 0 & 1 & 0 \\
0 & 1 & 0 & 0 \\
1 & 0 & 0 & 1
\end{array}\right)
$$

This shows the uniqueness part of the lemma. To show existence, we need to show that $\theta$ as given above is domestic, since it is clearly not point-domestic nor line-domestic. Also, one can check with an elementary calculation that $\theta$ does not fix any point of $\Gamma$.

One sees that $\theta^{2}$ fixes $\pi$ pointwise, and it also fixes all lines through the point $(0,1,1,0)$. Hence, since $\theta$ fixes exactly three lines through $(0,1,1,0)$ in $\pi$, and also at least one line off $\pi$ (for instance the line $\langle(0,1,1,0),(\epsilon, 0,0,1)\rangle$ ), it fixes exactly seven lines through $(0,1,1,0)$, and these seven lines form a Baer subplane in the projection from $(0,1,1,0)$. It follows that every point $x$ of $\mathrm{AG}(3,4)$ is contained in at least one plane $\beta$ through $(0,1,1,0)$ and fixed under $\theta$. Suppose that $x$ does not lie on one of the two affine fixed lines of $\beta$. Let $o_{1}$ and $o_{2}$ be the two points of $\mathcal{O}$ in $\beta$. Consider the line $x o_{1}$. Then both $\left(x o_{1}\right)^{\theta}$ and $\left(x o_{1}\right)^{\theta^{-1}}$ contain $o_{2}$ and must meet $x o_{1}$ in one of the two points of $x o_{1}$ not lying on a fixed line, as otherwise this intersection point would be fixed under $\theta$, a contradiction. But it is easy to see that both possibilities lead to $x^{\theta}$ being collinear to $x$ in $\Gamma$, since $x x^{\theta}$ contains either $o_{1}$ or $o_{2}$. Hence if $x^{\theta}$ is opposite $x$, then $x$ is incident with a fixed line $K$ of $\mathrm{AG}(3,4)$ through $(0,1,1,0)$. But then, for an arbitrary point $o$ in $\mathcal{O}$, the line $x o$ intersects the line $x^{\theta} o^{\theta}$, since both are contained in the same plane $\langle x, o,(0,1,1,0)\rangle$. This shows the assertion.

Now Theorem 2.1 is completely proved.

Remark 4.5 In the last paragraph of the previous proof, we established that $\theta^{2}$ is a translation in $\operatorname{AG}(3,4)$ with center $(0,1,1,0)$ at infinity. Since this point is not in the hyperoval, $\theta^{2}$ maps every flag to an opposite one. Hence $\theta^{2}$ is an involution without fixed elements. This is rather rare for finite generalized quadrangles, as this can never happen in any finite classical generalized quadrangle. Indeed, in this case the parameters $s$ and $t$ are not relatively prime, and it is noted in [8] that under this assumption, a result of Benson [3] implies that every involution has fixed elements. Our example shows the necessity of 
that assumption (in fact, an analogous example exists for all generalized quadrangles of type $T_{3}^{*}(O)$, with $O$ a hyperoval in a finite projective plane; for the precise definitions, see $[6])$.

It can also be shown directly from the data gathered in Section 3 that the square of any exceptional domestic collineation without fixed elements maps every flag onto an opposite one.

Acknowledgment. We would like to thank the anonymous referee for various interesting remarks that improved our paper.

\section{References}

[1] A. Bichara, F. Mazzocca \& C. Somma, On the classification of generalized quadrangles in a finite affine space AG $\left(3,2^{h}\right)$, Boll. Un. Mat. Ital. B17 (1980), 298-307.

[2] P. Abramenko \& K. Brown, Automorphisms of non-spherical buildings have unbounded displacement, Innov. Incidence Geom. 10 (2009), 1-13.

[3] C. T. Benson, On the structure of generalized quadrangles, J. Algebra 15 (1970), 443-454.

[4] R. Gramlich, Developments in finite Phan theory, Innov. Incidence Geom. 9 (2009), 123-175.

[5] B. Leeb, A characterization of irreducible symmetric spaces and Euclidean buildings of higher rank by their asymptotic geometry, Bonner Mathematische Schriften [Bonn Mathematical Publications] 326, Universität Bonn, Mathematisches Institut, Bonn, 2000 .

[6] S. E. Payne \& J. A. Thas, Finite Generalized Quadrangles, Research Notes in Mathematics 110, Pitman Advanced Publishing Program, Boston/London/Melbourne, 1984; 2nd edition, European Mathematical Society, EMS Series of Lectures in Mathematics, 2009.

[7] B. Temmermans, Dualities and Collineations of Projective and Polar spaces and of Related Geometries, Ph.D.-Thesis, Ghent University, 2010.

[8] B. Temmermans, J. A. Thas \& H. Van Maldeghem, On collineations and dualities of finite generalized polygons, Combinatorica 29 (2009), 569-594. 
[9] B. Temmermans, J. A. Thas \& H. Van Maldeghem, Domesticity in projective spaces, to appear in Innov. Incidence Geom.

[10] B. Temmermans, J. A. Thas \& H. Van Maldeghem, Collineations of polar spaces with restricted displacements, to appear in Des. Codes Cryptogr.

[11] J. A. Thas, 4-gonal subconfigurations of a given 4-gonal configuration, Rend. Accad. Naz. Lincei 53 (1972), $520-530$.

[12] J. A. Thas, A remark concerning the restriction on the parameters of a 4-gonal configuration, Simon Stevin 48 (1974), $65-68$.

[13] J. Tits \& R. Weiss, Moufang polygons, Springer-Verlag, 2002.

[14] H. Van Maldeghem, Generalized Polygons, Birkhäuser Verlag, Basel, Boston, Berlin, Monographs in Mathematics 93, 1998. 